\title{
Correction of systematic behaviour in topographical surface analysis
}

Quagliotti, Danilo; Baruffi, Federico; Tosello, Guido; Gasparin, S.; Annoni, M.; Parenti, P.; Sobiecki, Rene; Hansen, Hans Nørgaard

\section{Published in:}

Proceedings of the 11th International Conference on Multi-Material Micro Manufacture (4M2016) : co-organised with 10th International Workshop on Microfactories (IWMF2016)

Link to article, DOI:

10.3850/978-981-11-0749-8 738

Publication date:

2016

Document Version

Publisher's PDF, also known as Version of record

Link back to DTU Orbit

Citation (APA):

Quagliotti, D., Baruffi, F., Tosello, G., Gasparin, S., Annoni, M., Parenti, P., Sobiecki, R., \& Hansen, H. N. (2016). Correction of systematic behaviour in topographical surface analysis. In Proceedings of the 11th International Conference on Multi-Material Micro Manufacture (4M2016): co-organised with 10th International Workshop on Microfactories (IWMF2016) (pp. 277-280). Research Publishing Services.

https://doi.org/10.3850/978-981-11-0749-8 738

\section{General rights}

Copyright and moral rights for the publications made accessible in the public portal are retained by the authors and/or other copyright owners and it is a condition of accessing publications that users recognise and abide by the legal requirements associated with these rights.

- Users may download and print one copy of any publication from the public portal for the purpose of private study or research.

- You may not further distribute the material or use it for any profit-making activity or commercial gain

- You may freely distribute the URL identifying the publication in the public portal 


\title{
Correction of systematic behaviour in topographical surface analysis
}

\author{
D. Quagliotti', F. Baruffi 1,2, G. Tosello1', S. Gasparin ${ }^{3}$, M. Annoni $^{2}$, \\ P. Parenti ${ }^{2}$, R. Sobiecki ${ }^{1}$, H. N. Hansen ${ }^{1}$ \\ 11 Technical University of Denmark, Department of Mechanical Engineering, Kgs. Lyngby, Denmark \\ 2 Politecnico di Milano, Dipartimento di Ingegneria Meccanica, Milan, Italy \\ 3 The LEGO Group, Billund, Denmark
}

\begin{abstract}
Four specimens in the sub-micrometre range and with different polishing were topographically investigated in five areas over their respective surfaces. Uncertainties were evaluated with and without correction for systematic behaviour and successively analysed by a design of experiment (DOE). Results showed that the correction for systematic behaviour allowed for a lower value of the estimated uncertainty when the correction was adequate to completely recognise the systematic effects. If not, the correction can produce an overestimation of the uncertainty.
\end{abstract}

Keywords: systematics, systematic effects, surface analysis, topography, DOE.

\section{Introduction}

A manufactured component normally interacts with the environment, where it is intended to be used, through its surface. It is an interface where, as the technology progresses, more and more complex functionalities are resolved. As a consequence, the interest for three dimensional areal characterization is increasing [1-2].

In this context, optical instruments appear more adequate for functionality investigations [2]. Nevertheless, regardless the instrument used, the influence of the measuring process on the sought parameters of the topographical characterisation should be kept below reasonable limits [3].

In the following, an investigation of four specimens in the sub-micrometre range and with different polishing of their respective surfaces is described. The aim was to investigate if the evaluation of the uncertainty can be optimized to the variability of a surface with a control over the instrument influence. At this purpose, the uncertainties were evaluated with and without correction for systematic behaviours and successively analysed by a design of experiment (DOE).

Table 1

Specifications of the specimens under investigation and nominal roughness intervals

\begin{tabular}{lll}
\hline Sample & Surface finish & $\begin{array}{l}\text { Nominal Ra interval } \\
(\mathrm{nm})\end{array}$ \\
\hline$T 1$ & $\begin{array}{l}\text { Diamond buff } \\
\text { (grade 15) }\end{array}$ & $50-76$ \\
& 320 Grit paper & $229-254$ \\
$T 2$ & 400 Stone & $635-711$ \\
$T 3$ & 400 Dry blast & $254-305$ \\
$T 4$ & (glass bead 11) & \\
\hline
\end{tabular}

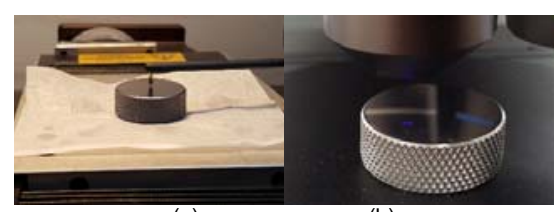

(a) (b)

Fig. 1. Example of investigated tools. (a): sample measured by contact instrument (Talysurf 50 ). (b): sample measured by confocal microscope (Olympus Lext)

\subsection{Specimens under investigation}

The examined specimens are four steel components of cylindrical shape and height about $1 \mathrm{~cm}$. They have polished flat surfaces with nominal diameter of $2.54 \mathrm{~cm}$ (two examples are in Fig. 1). Other nominal characteristics are reported in Table 1.

\section{Measurements}

The specimens were initially measured by a calibrated stylus instrument (Taylor-Hobson Talysurf, FTS) [4], performing 3D measurements in the centre of the surface of each sample under evaluation.

Table 2

Average $S a$ and $S q$ roughness parameters measured by reference contact instrument ${ }^{\mathrm{a}}$

\begin{tabular}{lll}
\hline Sample & SaFTS $(\mathrm{nm})$ & SqFTS $(\mathrm{nm})$ \\
\hline T1 & 48 & 61 \\
$T 2$ & 133 & 179 \\
T3 & 232 & 313 \\
T4 & 510 & 646 \\
\hline
\end{tabular}

a The expanded uncertainty stated for the contact instrument in the calibration certificate [4] is $10 \mathrm{~nm}$, for $R a$ values up to $229 \mathrm{~nm}$, and $24 \mathrm{~nm}$, for $R a$ values up $604 \mathrm{~nm}$. 


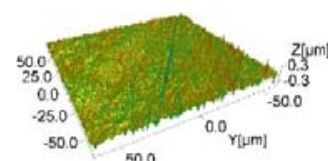

(a)

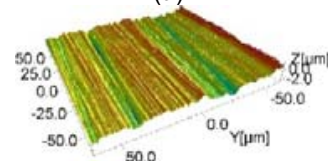

(c)

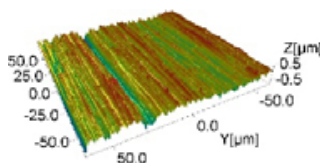

(b)

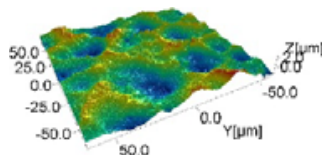

(d)
Fig. 2. Three dimensional view of acquired surfaces Examples of (a): T1; (b): T2; (c): T3; (d): T4.

An evaluation area $4 \mathrm{~mm} \times 10 \mathrm{~mm}$ was measured as 8200 pixels along the $x$-axis and 21 profiles along the $y$-axis. Such results were considered the reference for optical instrument measurements and used for correcting discrepancies in order to establish the traceability [5-6]. Averages of the reference results are in Table 2.

\subsection{Optical measurements}

Optical measurements were performed by Olympus Lext confocal microscope (magnification 100x: numerical aperture 0.95 , vertical resolution $10 \mathrm{~nm}$, pixel size $31.5 \mathrm{~nm} \times 31.5 \mathrm{~nm}$ ).

Considering the surfaces under evaluation of circular shape, the centre of each circle was defined as the origin $(0,0)$ of a local reference system, integral to the samples. Each sample was oriented on the stage of the instrument so that the laser scanning movement was orthogonal to the dominant texture (when it was possible to recognise a dominant texture). Hence, the direction of the laser scanning movement was chosen to identify the $y$-axis. The $x$ axis was set in order to have a right-handed reference system.

With reference to the local reference system, measurements were performed, in the central position $(0,0)$, and in the peripheral positions $(x, y),(x,-y),(-x, y)$, $(-x,-y)$, where $|x|=|y|=6.5 \mathrm{~mm}$.

\section{Processing}

The investigation was carried out inspecting for amplitude variations by $S q$ areal parameter. Data extraction was performed by [7], after correcting for the least square plane. No filter was used. Examples of acquired surfaces, one for each type of polishing, are in Fig. 2 (3D view). The data were successively examined for outliers by Chauvenet's criterion.

Table 3

Results of the surface analysis in the central area of the surfaces. Data corrected for systematic behaviour: $q$ is the slope of the model equation. $U$ is the expanded uncertainty. The reproducibility contributor $u_{R e p r}$ is also given. Values are in nanometre.

Sample $q(0,0) S q(0,0) u_{R e p r}(0,0) \cup(S q(0,0)) \cup(S q(0,0))$

\begin{tabular}{llllll}
\hline T1 & 0.8 & 49 & 0.6 & 28 & 28 \\
$T 2$ & 0.9 & 160 & 3.6 & 32 & 32 \\
$T 3$ & 1.2 & 388 & 3.1 & 43 & 43 \\
$T 4$ & 0.9 & 576 & 3.1 & 74 & 74 \\
\hline
\end{tabular}

Eventually, the uncertainty was evaluated after correcting for systematic behaviours. An uncertainty evaluation was also assessed without any systematics' correction. In both cases, traceability was established adjusting the optical measurements in agreement with the contact reference [5-6].

\subsection{Correction for systematic behaviour and uncertainty evaluation}

The correction for systematics was carried out by a least square fit of the optical measurements as function of the reference measurements. The model equation found consistent for best fitting the experimental data was a straight line with null constant term, i.d.:

$$
S q=q \cdot S q F T S
$$

Values of $q$ are in Table 3 and Table 5. Eq. 1 is the calibration equation of the optical measurements as a function of the reference. Inverting Eq. 1, it allows to estimate the reference values from optical ones.

In the case of no correction, traceability was established considering the deviation of the average of the optical measurements from the average of the references (Table 4 and Table 6).

In both cases, the evaluated uncertainty was in agreement with [8].

For the uncertainty of the best fit, the contributors considered were: 1) the standard uncertainty for the reference instrument, obtained dividing by 2 the expanded uncertainty stated in the calibration certificate [4]; 2) the reproducibility of the optical measurements; 3 ) the standard deviation of the coefficient of the model equation in the best fit regression; 4) the reproducibility of the best fit regression (see Table 3 for $(0,0)$ ).

When the correction was not performed, contributors to the uncertainty were: 1 ) the standard uncertainty for the reference instrument, obtained dividing by 2 the expanded uncertainty stated in the calibration certificate [4]; 2) the reproducibility of the optical measurements (see Table 4 for $(0,0)$ ); 3 ) the standard deviation of the optical measurements; 4 ) the standard deviation of the reference measurements.

Regarding the standard uncertainty of the reference instrument, it should be noted that two different values were available:

- $10 \mathrm{~nm}$, for Ra values up to $229 \mathrm{~nm}$. This value was related to the specimens $T 1, T 2$ and $T 3$

- $24 \mathrm{~nm}$, for Ra values up $604 \mathrm{~nm}$. This value was related to the specimen $T 4$.

Table 4

Results of the surface analysis in the central area of the surfaces. Data not corrected for systematic behaviour: no model equation coefficient is specified in this case $(q=0)$. $U$ is the expanded uncertainty. The reproducibility contributor $u_{R e p r}$ is also given. Values are in nanometre.

Sample $q(0,0) S q(0,0) u_{\operatorname{Repr}}(0,0) \cup(S q(0,0)) \cup(S q(0,0))$

\begin{tabular}{llllll}
\hline T1 & 0 & 49 & 0.5 & 35 & 35 \\
T2 & 0 & 160 & 3.3 & 36 & 36 \\
T3 & 0 & 388 & 2.5 & 35 & 35 \\
T4 & 0 & 576 & 3.0 & 84 & 84 \\
\hline
\end{tabular}


Table 5

Results of the surface analysis in the peripheral areas of the surface. Data corrected for systematic behaviour: $q$ is the slope of the model equation. $U$ is the expanded uncertainty. Values are in nanometre.

\begin{tabular}{lllll}
\hline Sample & $q(x, y)$ & $S q(x, y)$ & $\Delta S q(x, y)$ & $U(S q(x, y))$ \\
\hline$T 1$ & 0.8 & 47 & -2 & 28 \\
$T 2$ & 1.0 & 176 & 16 & 35 \\
$T 3$ & 1.3 & 407 & 19 & 46 \\
$T 4$ & 0.8 & 491 & -85 & 65 \\
\hline
\end{tabular}

\begin{tabular}{lllll}
\hline Sample & $q(x,-y)$ & $S q(x,-y)$ & $\Delta S q(x,-y)$ & $U(S q(x,-y))$ \\
\hline$T 1$ & 0.8 & 48 & -1 & 28 \\
$T 2$ & 1.1 & 193 & 33 & 38 \\
$T 3$ & 1.0 & 306 & -81 & 36 \\
T4 & 0.9 & 569 & -7 & 74 \\
\hline
\end{tabular}

\begin{tabular}{lllll}
\hline Sample & $q(-x, y)$ & $S q(-x, y)$ & $\Delta S q(-x, y)$ & $U(S q(-x, y))$ \\
\hline T1 & 0.7 & 45 & -5 & 26 \\
T2 & 0.9 & 154 & -6 & 30 \\
T3 & 0.7 & 223 & -165 & 25 \\
T4 & 0.8 & 525 & -51 & 68 \\
\hline
\end{tabular}

\begin{tabular}{lllll}
\hline Sample & $q(-x,-y)$ & $S q(-x,-y)$ & $\Delta S q(-x,-y)$ & $U(S q(-x,-y))$ \\
\hline T1 & 0.9 & 53 & 4 & 32 \\
$T 2$ & 0.8 & 140 & -20 & 28 \\
$T 3$ & 0.7 & 221 & -166 & 25 \\
$T 4$ & 0.9 & 594 & 18 & 78 \\
\hline
\end{tabular}

Successively, the uncertainty contributors were combined by applying the usual law for the propagation of the uncertainties.

Finally, the expanded uncertainty was evaluated as the confidence interval of $95 \%$. The relative coverage factor was calculated using the t-distribution with degrees of freedom evaluated by the WelchSatterthwaite formula (coverage factor in the range 2.08-2.20).

\section{Results}

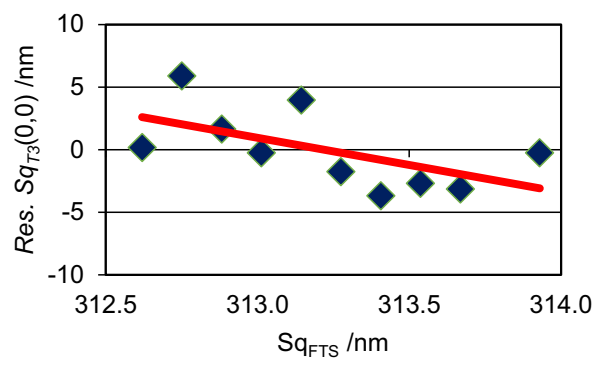

Fig. 3. Residuals of $S q(0,0)$ after systematics' correction for sample T3. The red straight line is the tendency.
Table 6

Results of the surface analysis in the peripheral areas of the surface. Data not corrected for systematic behaviour: no model equation coefficient is specified in this case $(q=0)$. $U$ is the expanded uncertainty. Values are in nanometre.

\begin{tabular}{|c|c|c|c|}
\hline Sample & $q(x, y)$ & $S q(x, y)$ & $U(S q(x, y))$ \\
\hline T1 & 0 & 47 & 35 \\
\hline$T 2$ & 0 & 176 & 35 \\
\hline T3 & 0 & 407 & 36 \\
\hline T4 & 0 & 491 & 85 \\
\hline Sample & $q(x,-y)$ & $S q(x,-y)$ & $U(S q(x,-y))$ \\
\hline$T 1$ & 0 & 48 & 35 \\
\hline$T 2$ & 0 & 193 & 35 \\
\hline T3 & 0 & 306 & 37 \\
\hline T4 & 0 & 569 & 84 \\
\hline Sample & $q(-x, y)$ & $S q(-x, y)$ & $U(S q(-x, y))$ \\
\hline$T 1$ & 0 & 45 & 36 \\
\hline$T 2$ & 0 & 154 & 35 \\
\hline T3 & 0 & 223 & 35 \\
\hline T4 & 0 & 525 & 83 \\
\hline Sample & $q(-x,-y)$ & $S q(-x,-y)$ & $U(S q(-x,-y))$ \\
\hline T1 & 0 & 53 & 36 \\
\hline$T 2$ & 0 & 140 & 35 \\
\hline T3 & 0 & 221 & 35 \\
\hline T4 & 0 & 594 & 85 \\
\hline
\end{tabular}

Average results and evaluated expanded uncertainties for the data corrected for systematic behaviour are in Table 3, for the measurements in the central area of the surfaces, and in Table 5, for the measurements in the peripheral areas. In the same tables, the values for the coefficient $q$ of the model equation are also given. Furthermore, in Table 5, the deviations $\Delta S q$ of the averages of the peripheral areas with respect to the averages in the central area of the surfaces are indicated. Deviations are defined as

$$
\Delta S q( \pm x, \pm y)=S q( \pm x, \pm y)-S q(0,0)
$$

Analogous average results and evaluated expanded uncertainties for the data without correction are in Table 4 and Table 6 respectively for the area in $(0,0)$ and for the other peripheral areas. No model equation was fitted in this circumstance. This condition is indicated in the tables as $q=0$.

\section{Discussion}

Inspecting the mean values of the measurements in the central area of the specimens' surfaces, reported in Table 3 and Table 4, it can be noted that they do not show any variation whether the correction for systematics was performed or not. In other words, in the study case examined, the correction of 


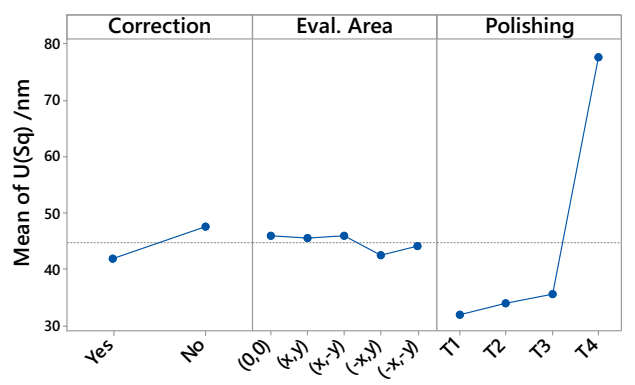

Fig. 5. Main effects plot of the DOE of the expanded uncertainty evaluated in the different areas of measurement, with and without systematics' correction.

systematics did not influence the mean values.

Analysing the uncertainties, instead, larger values are especially shown when the correction of systematics is not performed. Exception is the sample T3 which resulted in a larger expanded uncertainty evaluated after the correction of the systematics.

In Fig. 3, the inspection of the residuals of the regression, related to the sample $T 3$ and used for correcting the systematic behaviour, clearly reveals a residual tendency. In other words, the regression is not able to correct for all the systematics, despite a coefficient of determination of 0.999 and a significance of the regression coefficient over 200.

Indeed, the systematic behaviour cannot always be completely recognised. In such situations only a partial compensation of the systematic error can be achieved. Therefore, the unrecognised systematic effect is a source of bias which may have non-zero expected value and components of uncertainty that may increase the overall evaluated uncertainty [9].

Similar considerations can be done inspecting the mean values of the measurements in the peripheral areas reported Table 5 and Table 6 . In particular, the regression in $T 3(x, y)$ and in $T 2(x,-y)$ was not suitable to correct the systematic behaviour, resulting in an increased evaluated uncertainty.

A DOE was performed on the expanded uncertainties evaluated considering as effects under examination: 1) the polishing type; 2 ) the evaluation areas where the measurements were performed; 3 ) the correction of systematics.

From the examination of the main effects (Fig. 4), it can be noted that, in average:

1. The evaluated expanded uncertainty is lower when the systematics are corrected.

2 . The lowest uncertainty was stated for areas $(-x, y)$ and $(-x,-y)$. This result is influenced by the choice of the measured area for the reference. To avoid this effect and have a better estimation of the uncertainty the reference and the optical measurements should be performed and then matched over the same areas.

3. The lowest uncertainty was stated for 'Diamond buff' polishing, whilst the highest was for the 'Dry blast' polishing. Nonetheless, the last is an obvious result influenced by a higher uncertainty available in the calibration certificate for the roughness range of 'Dry blast' specimen.

The analysis of the interactions reported in Fig. 5

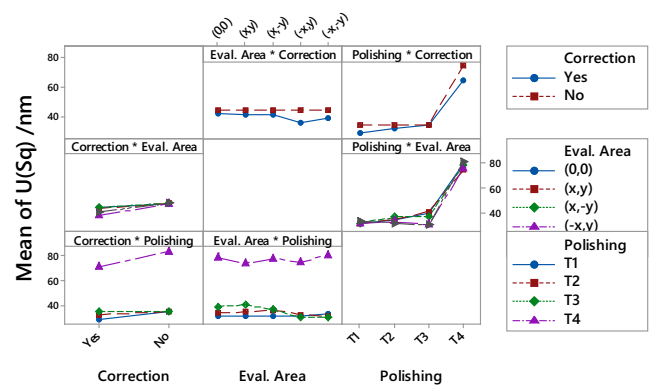

Fig. 4. Interaction plot of the DOE of the expanded uncertainty evaluated in the different areas of measurement, with and without systematics' correction.

confirms what already noticed. In average:

1. The higher uncertainty evaluated when the systematics are not corrected is independent from the evaluation area.

2. A lower uncertainty can be obtained for different types of polishing when systematics are corrected (exception is T3; no influence was noticed).

3. The evaluated uncertainty is to be considered not uniform depending on the areas of evaluation and polishing of the surfaces.

\section{Conclusions}

In conclusion, the investigation evidenced that, in the specific study case investigated, the correction of systematic behaviour in the measurement allowed to evaluate a lower expanded uncertainty, regardless of the type of specimen or evaluation area considered. Nevertheless, when the regression was not adequate to completely recognise the systematic effects, the correction resulted in an overestimation of the uncertainty.

\section{References}

[1] D. J. Whitehouse, Handbook of Surface and Nanometrology, IOP Publishing Ltd: 2003,1150.

[2] R. Leach Characterisation of Areal Surface Texture, R. Leach editor: 2013, 355.

[3] A. Weckenmann et al. "Acceptance of processes: do we need decision rules?" Precision Engineering, 2000; 24: 264-269.

[4] CGM (Center for Geometrical Metrology) DANAK Cal. Cert. RUM11011 for Taylor Hobson Form Talysurf series 2 Inductive 50, 2011.

[5] D. Quagliotti et al., "A Method for Dimensional and Surface Optical Measurements Uncertainty Assessment on Micro Structured Surfaces Manufactured by Jet-ECM," Proc. of the 4M/ICOMM2015 Conf.: 2015, 554-7.

[6] D. Quagliotti et al., "Optical micro-metrology of structured surfaces micro-machined by jet-ECM," Proc. of the $15^{\text {th }}$ euspen int. conf.: 2015, 167-8.

[7] SPIPTM, v. 6.5.2, Image Metrology A/S, www.imagemet.com.

[8] BIPM, Joint Committee for Guides in Metrology JCGM 100:2008.

[9] F. Pavese et al., "Data modeling for metrology and testing in measurement science" $F$. Pavese and A. B. Forbes ed., Birkhäuser: 2009, 499. 